\title{
CHARACTERIZATIONS OF POWER DISTRIBUTIONS VIA MOMENTS OF ORDER STATISTICS AND RECORD VALUES
}

Abstract. Power distributions can be characterized by equalities involving three moments of order statistics. Similar equalities involving three moments of $k$-record values can also be used for such a characterization. The case of samples with random sizes is also considered.

1. Introduction. Too and Lin [8] have given a characterization of the uniform distribution by an equality involving only two moments of order statistics. We extend that result to power distributions. Moreover, we give a characterization of power distributions in terms of moments of $k$-record values. In Sections 4 and 5 we treat the characterization problem when sample sizes are random (cf. [1], [6], [9]).

2. A characterization of power distributions. Let $X_{k: n}$ be the $k$ th smallest order statistic of a random sample $\left(X_{1}, \ldots, X_{n}\right)$ from a distribution $F$. Let $m$ be a negative integer. We start with the problem of characterizing the power distribution function $F$ defined as follows (cf. [1]):

$$
F(x)=1-(1+m x)^{-1 / m}, \quad x \in(0,-1 / m) .
$$

THEOREM 1. With the above notation suppose that $E X_{k: n}^{2}<\infty$ for some pair $(k, n)$. Then the equality

$$
\begin{aligned}
E X_{k: n}^{2}-\frac{2}{m}\left[\frac{n_{[k]}}{(n-m)_{[k]}} E X_{k: n-m}-E X_{k: n}\right] \\
+\frac{1}{m^{2}}\left[\frac{n_{[k]}}{(n-2 m)_{[k]}}-\frac{2 n_{[k]}}{(n-m)_{[k]}}+1\right]=0,
\end{aligned}
$$

where $n_{[k]}=n(n-1) \ldots(n-k+1)$, holds iff $F$ is given by $(2.1)$.

1991 Mathematics Subject Classification: Primary 62E10, 60E99.

Key words and phrases: sample, record values, power, uniform distributions. 

that

$$
E X_{k: n}^{l}=\frac{n !}{(k-1) !(n-k) !} \int_{0}^{1}\left(F^{-1}(t)\right)^{l} t^{k-1}(1-t)^{n-k} d t, \quad l \geq 1,
$$

we see that $E\left|X_{k: n}\right|<\infty$, and $E\left|X_{k: n-m}\right|<\infty$. Furthermore, when $F$ is given by $(2.1)$, we find that

$$
\begin{aligned}
E X_{k: s}= & \frac{k}{m}\left(\begin{array}{l}
s \\
k
\end{array}\right)[B(k, s-m-k+1) \\
& -B(k, n-k+1)], \quad n \leq s \leq n-m .
\end{aligned}
$$

and

$$
\begin{aligned}
E X_{k: n}^{2}= & \frac{k}{m^{2}}\left(\begin{array}{l}
n \\
k
\end{array}\right)[B(k, n-k-2 m+1)-2 B(k, n-k-m+1) \\
& +B(k, n-k+1)],
\end{aligned}
$$

where $B(a, b)$ is the Beta function, and so (2.2) holds true.

Conversely, assume that (2.2) holds. Applying (2.3) we see that (2.2) can be written as

$$
\int_{0}^{1}\left(F^{-1}(t)-\frac{(1-t)^{-m}-1}{m}\right)^{2} t^{k-1}(1-t)^{n-k} d t=0,
$$

which implies that $F(x)$ is given by $(2.1)$.

When $m=-1$ Theorem 1 reduces to the following characterization of the uniform distribution.

Corollary 1 (cf. [8]). Let $E X_{k: n}^{2}<\infty$ for some pair $(k, n)$. Then

$$
E X_{k: n}^{2}-\frac{2 k}{n+1} E X_{k+1: n+1}+\frac{k(k+1)}{(n+1)(n+2)}=0
$$

iff $F(x)=x$ on $(0,1)$.

In proving $\left(2.2^{\prime}\right)$ we use the equality

$$
(n-k) E X_{k: n}+k E X_{k+1: n}=n E X_{k: n-1}
$$

with $k=1$ (cf. [2]).

Using (2.4) and (2.5) we obtain the following characterizing conditions.

ThEOREM 1'. Under the assumptions of Theorem 1 the distribution function $F$ is given by (2.1) iff

$$
\begin{aligned}
& E X_{k: s}=\frac{1}{m}\left[\frac{s_{[k]}}{(s-m)_{[k]}}-1\right], \quad s=n, n-m, \\
& E X_{k: n}^{2}=\frac{1}{m^{2}}\left[\frac{n_{[k]}}{(n-2 m)_{[k]}}-2 \frac{n_{[k]}}{(n-m)_{[k]}}+1\right] .
\end{aligned}
$$


The same results can be derived for the distribution

$$
F(x)=1-(1+m x)^{-1 / m}, \quad x>0,
$$

where $m$ is a positive integer (cf. [1]). In this case (2.2) holds with $n-2 m-$ $k>0$.

\section{Characterizations in terms of moments of $k$-record values.}

Let $\left\{X_{n}, n \geq 1\right\}$ be a sequence of i.i.d. random variables with a common distribution function $F$. For a fixed integer $k \geq 1$ we define (cf. [3]) the sequence of $k$-record values as follows:

$$
Y_{n}^{(k)}=X_{L_{k}(n): L_{k}(n)+k-1}, \quad n \in \mathbb{N},
$$

where the sequence $\left\{L_{k}(n), n \geq 1\right\}$ of $k$-record times is given by $L_{k}(1)=1$, $L_{k}(n+1)=\min \left\{j: j>L_{k}(n), X_{j: j+k-1}>X_{L_{k}(n): L_{k}(n)+k-1}\right\}, n \in \mathbb{N}$.

A characterization of $F$ in (2.1) is contained in the following theorem.

Theorem 2. Let $\left\{X_{n}, n \geq 1\right\}$ be a sequence of i.i.d. random variables with a common distribution function $F$ such that $E\left|\min \left(X_{1}, \ldots, X_{k}\right)\right|^{2 p}<\infty$ for a fixed $k \geq 1$ and some $p>1$. Then $F$ is given by (2.1) iff

$$
\begin{aligned}
E\left(Y_{n}^{(k)}\right)^{2}-\frac{2}{m}\left[\left(\frac{k}{k-m}\right)^{n} E Y_{n}^{(k-m)}-E Y_{n}^{(k)}\right] & \\
+\frac{1}{m^{2}}\left(1-2\left(\frac{k}{k-m}\right)^{n}+\left(\frac{k}{k-2 m}\right)^{n}\right) & =0
\end{aligned}
$$

for $n=1,2, \ldots$

P r o of. Suppose that $F$ is given by (2.1). Then we have

$$
\begin{aligned}
E Y_{n}^{(k)} & =\frac{k^{n}}{(n-1) !} \int_{0}^{1} F^{-1}(t)[-\log (1-t)]^{n-1}(1-t)^{k-1} d t \\
& =\frac{1}{m}\left[\left(\frac{k}{k-m}\right)^{m}-1\right]
\end{aligned}
$$

and

$$
\begin{aligned}
E\left(Y_{n}^{(k)}\right)^{2} & =\frac{k^{n}}{(n-1) !} \int_{0}^{1}\left(F^{-1}(t)\right)^{2}[-\log (1-t)]^{n-1}(1-t)^{k-1} d t \\
& =\frac{k^{n}}{(n-1) !} \int_{0}^{1}\left[(1-t)^{-m}-1\right]^{2}[-\log (1-t)]^{n-1}(1-t)^{k-1} d t \\
& =\frac{k^{n}}{(n-1) ! m^{2}}\left[\frac{\Gamma(n)}{(k-2 m)^{n}}-\frac{2 \Gamma(n)}{(k-m)^{n}}+\frac{1}{k^{n}} \Gamma(n)\right] \\
& =\frac{1}{m^{2}}\left[\left(\frac{k}{k-2 m}\right)^{n}-2\left(\frac{k}{k-m}\right)^{n}+1\right]
\end{aligned}
$$




$$
\left(\frac{k}{k-m}\right)^{n} E Y_{n}^{(k-m)}=\frac{k^{n}}{m}\left(\frac{1}{(k-2 m)^{n}}-\frac{1}{(k-m)^{n}}\right),
$$

which establishes (3.1).

Conversely, assuming that (3.1) is satisfied we see that

$$
\int_{0}^{1}\left[F^{-1}(t)-\frac{(1-t)^{-m}-1}{m}\right]^{2}[-\log (1-t)]^{n-1}(1-t)^{k-1} d t=0 .
$$

Since the sequence $\left\{(-\log (1-t))^{n}, n \geq 1\right\}$, is complete in $L(0,1)$ (cf. [7]) we conclude that $F(x)$ is of the form (2.1).

TheOREM 2'. Under the assumptions of Theorem 2 the distribution function $F(x)$ is given by (2.1) for $k>2 m$ iff the following relations hold:

$$
\begin{aligned}
E Y_{n}^{(s)} & =\frac{1}{m}\left[\left(\frac{s}{s-m}\right)^{n}-1\right], \quad s=k, k-m, \\
E\left(Y_{n}^{(k)}\right)^{2} & =\frac{1}{m^{2}}\left[\left(\frac{k}{k-2 m}\right)^{n}-2\left(\frac{k}{k-m}\right)^{n}+1\right]
\end{aligned}
$$

for $n=1,2, \ldots$

Putting $m=-1$ we obtain the characterization results given in [6]. For $n=1, m=-1$ we obtain the result of Too and Lin [8].

Similar considerations lead to the analogous characterizations for the distribution (2.6). Namely, we have the following results.

TheOREM 3. Let $\left\{X_{n}, n \geq 1\right\}$ be a sequence of i.i.d. random variables with a common distribution function $F$ such that $E\left|\min \left(X_{1}, \ldots, X_{k}\right)\right|^{2 p}<\infty$ for a fixed $k \geq 1$ and some $p>1$. Then $F(x)$ has the form (2.6) iff for $k-2 m>0$, where $m$ is a positive integer,

$$
\begin{aligned}
& E\left(Y_{n}^{(k)}\right)^{2}-\frac{2}{m}\left[\left(\frac{k}{k-m}\right)^{n} E Y_{n}^{(k-m)}-E Y_{n}^{(k-m-1)}\right] \\
&+\frac{1}{m^{2}}\left[1-2\left(\frac{k}{k-m}\right)^{n}+\left(\frac{k}{k-2 m}\right)^{n}\right]=0
\end{aligned}
$$

for $n=1,2, \ldots$

TheOREM 3'. Under the assumptions of Theorem 3, the distribution function $F$ is given by (2.6) iff for $k-2 m>0$,

$$
\begin{aligned}
E Y_{n}^{(s)} & =\frac{1}{m}\left[\left(\frac{s}{s-m}\right)^{n}-1\right], \quad s=k, k-m, \\
E\left(Y_{n}^{(k)}\right)^{2} & =\frac{1}{m^{2}}\left[\left(\frac{k}{k-2 m}\right)^{n}-2\left(\frac{k}{k-m}\right)^{n}+1\right]
\end{aligned}
$$

for $n=1,2, \ldots$ 
Letting $m=1$ we obtain the following characterization result.

Corollary 2. $F(x)=1-(1+x)^{-1}, x>0$, iff $E\left(Y_{n}^{(k)}\right)^{2}-2\left[\left(\frac{k}{k-1}\right)^{n} E Y_{n}^{(k-1)}-E Y_{n}^{(k)}\right]+1-2\left(\frac{k}{k-1}\right)^{n}+\left(\frac{k}{k-2}\right)^{n}=0$.

4. Characterizations by moments of randomly indexed order statistics. Let $X_{k: N}$ be the $k$ th smallest order statistics of a random sample $\left(X_{1}, \ldots, X_{N}\right)$ with common distribution function $F$, where $N$ is a random variable independent of $\left\{X_{n}, n \geq 1\right\}$ with a probability function $p(k)=$ $P[N=k], k=1,2, \ldots$ We write $P_{k}=P[N \geq k]$.

In this section we give a characterization for the distribution (2.1) in terms of moments of order statistics with a random index.

THEOREM 4. With the above notation, suppose that $E\left(X_{k: N}^{2} \mid N \geq k\right)$ $<\infty$ for some $k$ and a given probability function $p(\cdot)$ of $N$. Then

$$
\begin{aligned}
& E\left(X_{k: N}^{2} \mid N \geq k\right) \\
- & \frac{2}{m}\left[E\left(\frac{N_{[k]}}{(N-m)_{[k]}} X_{k: N-m} \mid N \geq k\right)-E\left(X_{k: N} \mid N \geq k\right)\right] \\
+ & \frac{1}{m^{2}}\left[E\left(\frac{N_{[k]}}{(N-2 m)_{[k]}} \mid N \geq k\right)-2 E\left(\frac{N_{[k]}}{(N-m)_{[k]}} \mid N \geq k\right)+1\right]=0,
\end{aligned}
$$

where $N_{[k]}=N(N-1) \ldots(N-k+1)$, iff $F$ is given by $(2.1)$.

Pr o of. Let $F^{-1}(t)=\inf \{x: F(x) \geq t, t \in(0,1)\}$. We have

$$
E X_{k: n}^{l}=\frac{n !}{(k-1) !(n-k) !} \int_{0}^{1}\left(F^{-1}(t)\right)^{l} t^{k-1}(1-t)^{n-k} d t, \quad l \geq 1 .
$$

Suppose that $F$ is given by (2.1). Since $N$ is independent of $\left\{X_{n}, n \geq 1\right\}$, from (2.3) we have

$$
\begin{aligned}
E\left(X_{k: N} \mid N \geq k\right)= & \frac{1}{m P_{k}} \sum_{n=k}^{\infty} \frac{n !}{(k-1) !(n-k) !} \\
& \times \int_{0}^{1}\left[(1-t)^{-m}-1\right] t^{k-1}(1-t)^{n-k} d t P[N=n] \\
= & \frac{1}{m} E\left(\left(\frac{N_{[k]}}{(N-m)_{[k]}} \mid N \geq k\right)-1\right),
\end{aligned}
$$




$$
\begin{aligned}
E\left(\frac{N_{[k]}}{(N-m)_{[k]}} X_{k: N-m} \mid N \geq k\right) \\
=\frac{1}{m P_{k}} \sum_{n=k}^{\infty} \frac{(n-m) !}{(k-1) !(n-k-m) !} \\
\quad \times \frac{n(n-1) \ldots(n-k+1)}{(n-m)(n-m-1) \ldots(n-m-k+1)} \\
\quad \times \int_{0}^{1}\left((1-t)^{-m}-1\right) t^{k-1}(1-t)^{n-m-k} d t P[N=n] \\
=\frac{1}{m}\left[E\left(\frac{N_{[k]}}{(N-2 m)_{[k]}} \mid N \geq k\right)-E\left(\frac{N_{[k]}}{(N-m)_{[k]}} \mid N \geq k\right)\right]
\end{aligned}
$$

and

$$
\begin{aligned}
& E\left(X_{k: N}^{2} \mid N \geq k\right) \\
& =\frac{1}{m^{2}}\left[E\left(\frac{N_{[k]}}{(N-2 m)_{[k]}} \mid N \geq k\right)-2 E\left(\frac{N_{[k]}}{(N-m)_{[k]}} \mid N \geq k\right)+1\right] .
\end{aligned}
$$

We see that (4.1) holds true.

Conversely, assume that (4.1) holds. It can be written as

$$
\sum_{n=k}^{\infty} \int_{0}^{1}\left(F^{-1}(t)-\frac{(1-t)^{-m}-1}{m}\right)^{2} t^{k-1}(1-t)^{n-k} d t P[N=n]=0,
$$

which implies that $F$ is given by (2.1).

Using (4.3)-(4.5) we have the following characterization conditions in terms of conditional moments of order statistics.

THEOREM 4'. Under the assumptions of Theorem 4 the distribution function $F$ is given by (2.1) iff

$$
\begin{gathered}
E\left(X_{k: N} \mid N \geq k\right)=\frac{1}{m}\left[E\left(\frac{N_{[k]}}{(N-m)_{[k]}} \mid N \geq k\right)-1\right] \\
E\left(\frac{N_{[k]}}{(N-m)_{[k]}} X_{k: N-m} \mid N \geq k\right) \\
\quad=\frac{1}{m}\left[E\left(\frac{N_{[k]}}{(N-2 m)_{[k]}} \mid N \geq k\right)-E\left(\frac{N_{[k]}}{(N-m)_{[k]}}\right)\right]
\end{gathered}
$$

and

$$
\begin{aligned}
E\left(X_{k: N}^{2} \mid N \geq k\right)= & \frac{1}{m^{2}}\left[E\left(\frac{N_{[k]}}{(N-2 m)_{[k]}} \mid N \geq k\right)\right. \\
& \left.-2 E\left(\frac{N_{[k]}}{(N-m)_{[k]}} \mid N \geq k\right)+1\right] .
\end{aligned}
$$


Corollary 3. Let $N$ be a random variable with probability function

$$
P[N=n]=\frac{\alpha \theta^{n}}{n}, \quad n=1,2, \ldots ; \alpha=-\frac{1}{\ln (1-\theta)}, \theta \in(0,1) .
$$

Then $X$ has the distribution (2.1) iff

$$
\begin{aligned}
E X_{1: N} & =\frac{1}{m}\left[\theta^{m}\left(1-\alpha \sum_{n=1}^{-m} \frac{\theta^{n}}{n}\right)-1\right], \\
E \frac{N}{N-m} X_{1: N-m} & =\frac{1}{m}\left[\theta^{2 m}-\theta^{m}-\theta^{2 m} \alpha \sum_{n=1}^{-2 m} \frac{\theta^{n}}{n}+\theta^{m} \alpha \sum_{n=1}^{-m} \frac{\theta^{n}}{n}\right], \\
E X_{1: N}^{2} & =\frac{1}{m^{2}}\left[\theta^{2 m}-2 \theta^{m}-\alpha \theta^{2 m} \sum_{n=1}^{-2 m} \frac{\theta^{n}}{n}+2 \theta^{m} \alpha \sum_{n=1}^{-m} \frac{\theta^{n}}{n}+1\right] .
\end{aligned}
$$

Remark. Putting $m=-1$ we obtain a characterization of the uniform distribution in terms of $X_{1: N}$, which after using the equality

$$
E X_{1: N}^{2}-E \frac{N}{N+1} X_{1: N}=\alpha E X_{1}+\left(1-\frac{1}{\theta}\right) E X_{1: N}
$$

leads to the result of [9], i.e.

$$
E X_{1: N}^{2}-2\left[\alpha E X+\left(1-\frac{1}{\theta}\right) E X_{1: N}\right]=-\alpha\left[\frac{3}{2}-\frac{1}{\theta}-\left(1-\frac{1}{\theta}\right)^{2} \ln (1-\theta)\right] .
$$

5. Characterizations via moments of randomly indexed record statistics

THEOREM 5. Let $Y_{N}^{(k)}$ be the kth record value, where $N$ is a positive integer-valued random variable independent of $\left\{X_{n}, n \geq 1\right\}$, and suppose that $E\left(Y_{N}^{(k)}\right)^{2}<\infty$. Then $F$ is given by (2.1) iff

$$
\begin{aligned}
E\left(Y_{N}^{(k)}\right)^{2}-\frac{2}{m} & {\left[E\left(\frac{k}{k-m}\right)^{N} Y_{N}^{(k-m)}-E Y_{N}^{(k)}\right] } \\
& +\frac{1}{m^{2}}\left[1-2 E\left(\frac{k}{k-m}\right)^{N}+E\left(\frac{k}{k-2 m}\right)^{N}\right]=0 .
\end{aligned}
$$

Pro of. Suppose that $F$ is given by (2.1). Since

$$
E\left(Y_{n}^{(k)}\right)^{l}=\frac{k^{n}}{(n-1) !} \int_{0}^{1}\left(F^{-1}(t)\right)^{l}[-\log (1-t)]^{n-1}(1-t)^{k-1} d t
$$

and $N$ and $\left\{X_{n}, n \geq 1\right\}$ are independent, it follows that

$$
\begin{aligned}
E Y_{N}^{(k)} & =\frac{1}{m} E\left[\left(\frac{k}{k-m}\right)^{N}-1\right], \\
E\left(\frac{k}{k-m}\right)^{N} Y_{N}^{(k-m)} & =\frac{1}{m}\left[E\left(\frac{k}{k-2 m}\right)^{N}-E\left(\frac{k}{k-m}\right)^{N}\right],
\end{aligned}
$$


which establishes (5.1).

$$
E\left(Y_{N}^{(k)}\right)^{2}=\frac{1}{m^{2}}\left[E\left(\frac{k}{k-2 m}\right)^{N}-2 E\left(\frac{k}{k-m}\right)^{N}+1\right],
$$

Assuming now that (2.1) is satisfied we see that

$$
\begin{aligned}
\sum_{n=1}^{\infty} \frac{k^{n}}{(n-1) !} \int_{0}^{1}\left(F^{-1}(t)\right. & \left.-\frac{(1-t)^{-m}-1}{m}\right)^{2} \\
& \times[-\log (1-t)]^{n-1}(1-t)^{k-1} d t P[N=n]=0 .
\end{aligned}
$$

Since the sequence $\left\{(-\log (1-t))^{n}, n \geq 1\right\}$ is complete in $L(0,1)$ (cf. [7]) it follows that $F(x)$ has the form (2.1).

Putting $m=-1$ we have the following characterization.

Corollary 4. $F(x)=x, x \in(0,1)$, iff

$$
\begin{aligned}
E\left(Y_{N}^{(k)}\right)^{2}+2\left(E\left(\frac{k}{k+1}\right)^{N}\right. & \left.Y_{N}^{(k+1)}-E Y_{N}^{(k)}\right) \\
& +E\left(\frac{k}{k+2}\right)^{N}-2 E\left(\frac{k}{k+1}\right)^{N}+1=0
\end{aligned}
$$

(cf. [5] with $m=1$ ).

COROllary 5. Let $N$ be a random variable with the probability function (4.7). Then $X$ has the distribution (2.1) iff

$$
\begin{aligned}
E\left(Y_{N}^{(k)}\right)^{2}- & \frac{2}{m}\left[E\left(\frac{k}{k-m}\right)^{N} Y_{N}^{(k-m)}-E Y_{N}^{(k)}\right] \\
& +\frac{1}{m^{2}}\left[1+2 \alpha \log \frac{k(1-\theta)-m}{k-m}-\alpha \log \frac{k(1-\theta)-2 m}{k-2 m}\right] .
\end{aligned}
$$

REMARK. $F(x)=x, x \in(0,1)$, iff

$$
\begin{aligned}
E\left(Y_{N}^{(k)}\right)^{2}+2(E & \left.\left(\frac{k}{k+1}\right)^{N} Y_{N}^{(k+1)}-E Y_{N}^{(k)}\right) \\
& +1+2 \alpha \log \frac{k(1-\theta)+1}{k+1}-\alpha \log \frac{k(1-\theta)+2}{k+2}=0 .
\end{aligned}
$$

Acknowledgements. The authors are grateful to the referee for useful comments which improved the presentation of the paper.

\section{References}

[1] M. Ahsanullah, Characteristic properties of order statistics based on random sample size from an exponential distribution, Statist. Neerlandica 42 (1988), 193-197.

[2] H. A. David, Order Statistics in Probability and Mathematical Statistics, Wiley, 1970. 
[3] W. Dziubdziela and B. Kopociński, Limiting properties of the kth record values, Zastos. Mat. 15 (1976), 187-190.

[4] Z. Grudzień and D. Szynal, Characterizations of distributions by moments of order statistics when the sample size is random, Appl. Math. (Warsaw) 23 (1995), 305-318.

[5] - - - Characterization of continuous distributions in terms of moments of extremal statistics, J. Math. Sci. 81 (1996), 2912-2936.

[6] - - - Characterizations of uniform and exponential distributions via moments of the kth record values randomly indexed, Appl. Math. (Warsaw) 24 (1997), 307-314.

[7] G. D. Lin, Characterizations of distribution via moments of order statistics: A survey and comparison of methods, in: Statistical Data Analysis and Inference, Y. Dodge (ed.), North-Holland, 1989, 297-307.

[8] Y. H. Too and G. D. Lin, Characterizations of uniform and exponential distributions, Statist. Probab. Lett. 7 (1989), 357-359.

[9] G. D. Lin and Y. H. Too, Characterization of distributions via moments of order statistics when sample size is random, Southeast Asian Bull. Math. 15 (1991), 139-144.

Department of Mathematics

Maria Curie-Skłodowska University

Pl. M. Curie-Skłodowskiej 1

20-031 Lublin, Poland

E-mail: szynal@golem.umcs.lublin.pl

Received on 7.5.1999;

revised version on 12.7.1999 